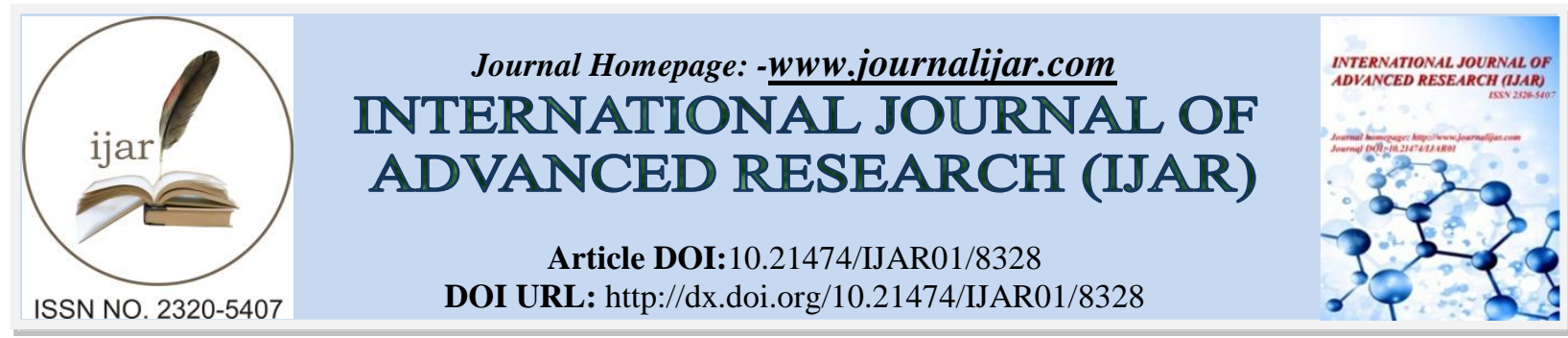

RESEARCH ARTICLE

\title{
NEXT-GENERATION SEQUENCING (NGS): OUR RESULTS OF MOLECULAR PATHOLOGY MALIGNANT SOLID TUMOR PANEL.
}

\author{
Ibrahim Halil Erdogdu. \\ Aydin Adnan Menderes University, Medical Faculty, Department of Pathology.
}

\section{Manuscript Info}

Manuscript History

Received: 04 November 2018

Final Accepted: 06 December 2018

Published: January 2019

Key words:-

NGS, cancer, mutation.

\begin{abstract}
Next-generation sequencing (NGS) has been widely used in cancer development, diagnosis and treatment in recent years. It offers new possibilities for the detection of mutations in malignant solid tumors. In this study, the results of malignant solid tumors with NGS were evaluated.

A total of 263 NGS cancer cases were included in the study. A total of 345 mutations were detected by the NGS 12 gene panel. The most common mutation was observed in NSCLC cases. The most common K-ras mutation was observed in all cancers. More than one mutation was detected in $71.8 \%$ of the cases. The mutations showing the most frequent accumulation are K-ras, PIK3CA mutations.

NGS, which is a fast and easy method, provides new possibilities in diagnosis, treatment and carcinogenesis in cancer cases. According to the study results, there were more than one mutation in malignant solid tumors. The selection of NGS panel should be based on the type of cancer and regional differences. In order to determine the effect of additional mutations on the treatment and prognosis in cancers with more than one mutation, studies should be conducted with a large series of cases.
\end{abstract}

Copy Right, IJAR, 2017,. All rights reserved.

\section{Introduction:-}

Next-generation sequencing (NGS) is a new technology product that has been introduced recently, allowing for sequencing millions of DNA fragments simultaneously. With this advanced technology, a new era has started compared to the the traditional methods that sequence relatively short DNA fragments. Because the traditional sequencing methods demand a large labour force and because they are costly, sequencing is performed with them only at defined regions of DNA (Damodaran S,et al.,2015). However, NGS allows for screening the whole genome in a shorter period of time and it is less costly. This revolutionary technology offers new options for the diagnosis and treatment of genetic diseases, infectious diseases, and cancer. Today, NGS results play a very important role in the diagnosis of cancer, in gaining insight into the mechanisms involved in the development of cancer, in the identification of prognostic indicators, and especially in the decision-making phase for administering targeted therapies (KoitzschU,et al., 2017). Currently, there are several target molecules that have been identified for personalized treatments in American and European guidelines (Kamps R, et al., 2017, Yu B, et al 2015). In order to identify these targets, the molecular profile of a tumour should be completely and reliably defined. Several various approaches have been defined for panel selection for NGS in cancer patients. Some cancer types (such as lung or

Corresponding Author:-Ibrahim Halil Erdogdu.

Address:-Aydin Adnan Menderes University, Medical Faculty, Department of Pathology. 
colorectal cancers), in which targeted treatments are indicated as options, are associated with specific mutations (Cancer Genome Atlas Research Network, 2014). However, creating a common panel in NGS for several types of cancer remains to be a challenge in overcoming the difficulties in panel selection. Approaches in the selection of general or customized panels will be more clearly defined as the island of knowledge in this area grows larger. It should also be noted that the selection of appropriate NGS panels depends on the number of regionally different types of mutations as they are observed in some types of cancers (D'Haene N,et al., 2018) For these reasons, it is important to determine mutation frequencies of patients with various types of cancer in different geographical regions. NGS data, which have been used in our department for about one year, was evaluated in this study in order to determine the distribution of common mutations in various types of cancer.

\section{Material AndMethod:-}

This study was retrospective study that approved by the Adnan Menderes University Faculty of Medicine Ethics Committee (No: 06/2019).

A total of 263 patients, for whom NGS panel requests were submitted to Adnan Menderes University Hospital, Pathology Laboratory, Molecular Pathology Unit between February 2018- December 2018 were included in the study. Formalin-fixed paraffin-embedded (FFPE) tissue samples of the patients were evaluated concomitantly with the respective previous pathology reports and hematoxylin and eosin (HE) stained sections. Materials of the patients included in the study were tested by using the GeneReader Platform NGS system (Qiagen, Hilden, Germany) as it has been described below:

\section{DNA Isolation:-}

Tumour areas were marked by pathologists and 2 sections with a thickness of 10 um were created from the tumour block. Automated DNA isolation was performed with the QIAcube device using GeneRead DNA FFPE Kit. After performing DNA quantification with the Qubit device, DNA concentration was checked with the Rotor-Gene Q instrument. Of a total of 263 isolated samples, all was quality controlled with qPCR, yielding a sufficient amount of products for the conduct of further processes. For the targeted enrichment step, a strict PCR protocol of 26-cycles was used for all samples to be processed with GeneReader workflows, according to the manufacturer's protocol.

\section{GeneReader Test and Sequencing:-}

In total, $40 \mathrm{ng}$ of each gDNA was used as a template for the QIAGEN Actionable Insight Tumor Panel. QIAGEN ATP analysis contains 330 amplicons of $16.7 \mathrm{~kb}$ associated with 773 unique variant positions in 12 prognostic and therapeutic genes (KRAS, NRAS, KIT, BRAF, PDGFRA, ALK, EGFR, ERBB2, PIK3CA, ERBB3, ESR1, and RAF1). Targeted amplicons were further processed to create a library for sequencing. The libraries were created using an automated protocol for QIAGEN GeneRead DNA Library Kit and QIAcube according to the manufacturer's instructions. Both PCR enriched DNA and GeneRead libraries were quantified using a QIAGEN QIAxcel Advanced System, complying with the manufacturer's instructions. Emulsion PCR and bead enrichment steps were performed in compliance with the manufacturer's instructions, using a GeneRead Clonal Amp Q Kit and an automated protocol in GeneRead QIAcube. After clonal amplification, the amplicon libraries were sequenced using QIAGEN GeneRead Sequencing Q Kit and an automated protocol in a GeneReader device.

\section{GeneReader Data Processing:-}

The QIAGEN Clinical Insight Analysis (QCI-A) software performed the secondary analysis of the FASTQ readings, which were generated by GeneReader with the alignment of the reference readings in the form of an interactive report with variant calling and allowing for the visualisation and the quality controlling of the array results. All comparable variants were identified with a QCI-A secondary analysis pipeline for ATP (ATPf) used for a 5\% allele fraction truncation for variant calling in the FFPE samples.

After examining the data validity, variants were transferred to the QCI interpretation (QCI-I) web interface, which enabled data interpretation for predefined variants. A report was generated, comprising a summary of the findings per sample for each variable identified based on the content of the QCI-I followed by the QIAGEN Knowledge Base with a direct link to the data source and listing the recommended treatment.

\section{Exons Tested in GeneReader:-}

KRAS (Exon 2, 3, 4, 5, 6)

NRAS (Exon 2, 3, 4) 
EGFR (Exon 2, 3, 4, 5, 6, 7, 8, 11, 12, 13, 14, 15, 16, 17, 18, 19, 20, 21, 22, 23, 24, 25)

C-KIT (Exon 1, 2, 4, 8, 9, 10, 11, 13, 14, 15, 17, 18, 20, 21)

BRAF (Exon 2, 3, 6, 7, 8, 11, 12, 15, 16, 17)

PDGFRA (Exon 2, 3, 4, 5, 6, 7, 11, 12, 13, 14, 15, 18, 23)

ALK (Exon 2, 3, 4, 5, 15, 18, 19, 20, 22, 23, 24, 25, 29)

ERBB2 (Exon 2, 3, 4, 5, 6, 8, 10, 11, 12, 13, 14, 15, 16, 17, 18, 19, 20, 22, 23, 24, 25, 26, 28)

ERBB3 (Exon 1, 2, 3, 4, 5, 6, 7, 8, 9, 10, 12, 22, 23, 24, 27)

ESR1 (Exon 1, 2, 3, 4, 6, 8, 9, 10)

RAF1 (Exon 1, 3, 4, 5, 6, 7, 13, 14, 16, 17, 18)

PIK3CA (Exon 2, 3, 5, 6, 7, 8, 9, 10, 19, 20, 21)

\section{Results:-}

Of a total of 263 samples tested; 92 were diagnosed with colorectal cancers (CRC), 127 with nonsmall cell lung cancers (NSCLC), 14 with thyroid cancers, 19 with malignant melanomas, and 11were diagnosed with GIST (Figure 1). The mean age was 52.4. in patients with CRC, 56.8 in NSCLC, 44,2 in thyroid cancer, 51.3 in malignant melanoma, and 49.2 in GIST. The most common mutation was Kras in CRC, EGFR in NSCLC, BRAF in malignant melanoma and thyroid cancer, and C-kit and PDGFRA in GIST. More than one mutation was observed in 189 of the patients. Of the patients with CRC, 34 had more than one mutation. The most common combination was the concomitant presence of K-ras and PIK3CA mutations. There were three or more mutations in three patients. Of the patients with NSCLC, 27 had more than one mutation. The most common combination was the concomitant presence of EGFR and PIK3CA mutations. Of the malignant melanoma patients, there was a concomitant presence of BRAF and EGFR mutations in 5 patients. The concomitant presence of C-kit and PDGFRA was identified in 9 patients with GIST. BRAF and K-ras mutations were concomitantly present in one patient with thyroid cancer. The mutations identified in the study patients are summarized in Table 1.

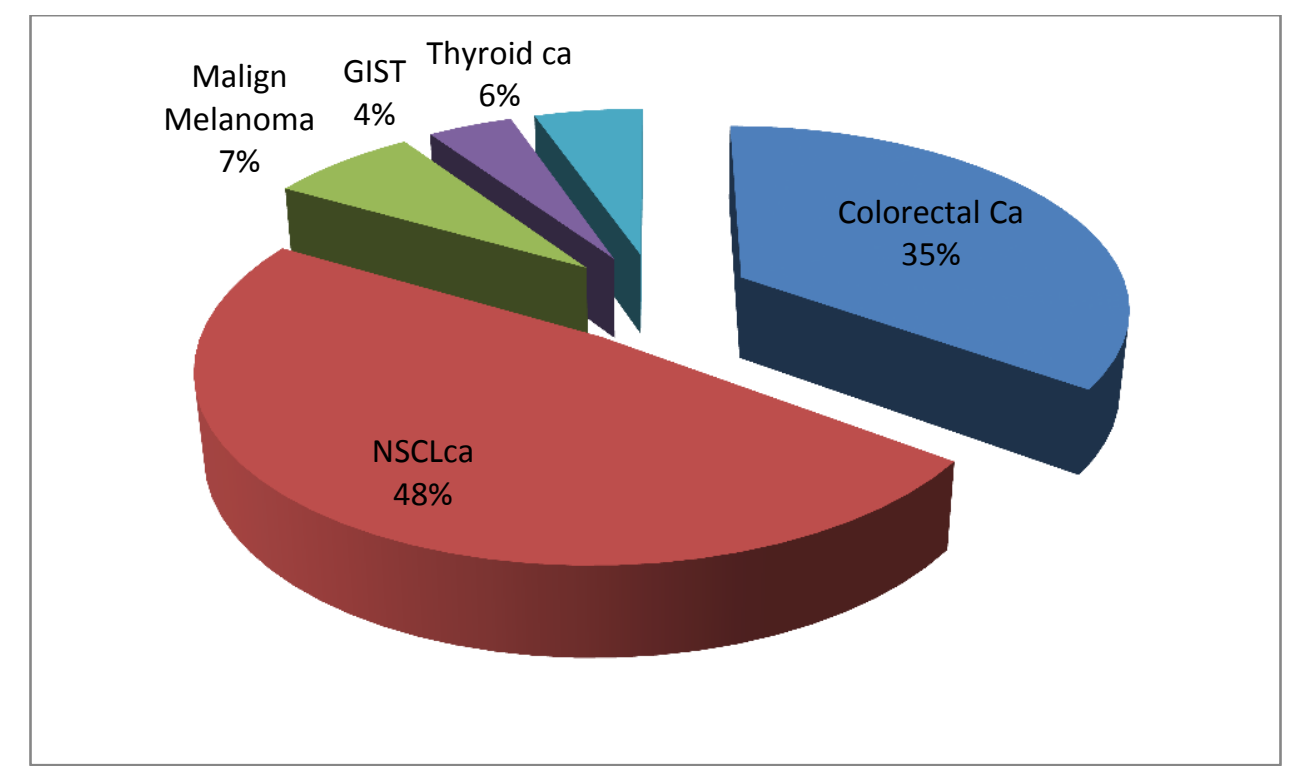

Figure 1:-Distribution of cancers in NGS. 
Table 1:-Types of mutations seen in cancers.

\begin{tabular}{|c|c|c|c|c|c|c|}
\hline & $\begin{array}{c}\text { COLORECTAL } \\
\text { CANCER }\end{array}$ & $\begin{array}{c}\text { NONSMALL } \\
\text { CELL LUNG } \\
\text { CA }\end{array}$ & $\begin{array}{c}\text { MALIGN } \\
\text { MELANOMA }\end{array}$ & $\begin{array}{c}\text { THYROID } \\
\text { CANCER }\end{array}$ & GIST & TOTAL \\
\hline KRAS & 63 & 46 & 2 & 4 & $\mathbf{0}$ & 115 \\
\hline Exon 2 & 43 & 38 & 1 & 0 & 0 & \\
\hline Exon 3 & 3 & 0 & 1 & 4 & 0 & \\
\hline Exon 4 & 15 & 0 & 0 & 0 & 0 & \\
\hline Exon 5 & 2 & 8 & 0 & 0 & 0 & \\
\hline NRAS & 2 & $\mathbf{0}$ & $\mathbf{0}$ & $\mathbf{0}$ & $\mathbf{0}$ & 2 \\
\hline Exon 2 & 2 & 0 & 0 & 0 & 0 & \\
\hline BRAF & 9 & 14 & 12 & 9 & 0 & 44 \\
\hline Exon 7 & 3 & 4 & 0 & 0 & 0 & \\
\hline Exon 11 & 0 & 2 & 0 & 0 & 0 & \\
\hline Exon 15 & 6 & 8 & 12 & 9 & 0 & \\
\hline EGFR & 2 & 34 & 8 & $\mathbf{0}$ & $\mathbf{0}$ & 44 \\
\hline Exon 13 & 0 & 3 & 2 & 0 & 0 & \\
\hline Exon 18 & 2 & 4 & 0 & 0 & 0 & \\
\hline Exon 19 & 0 & 11 & 0 & 0 & 0 & \\
\hline Exon 20 & 0 & 6 & 6 & 0 & 0 & \\
\hline Exon 21 & 0 & 10 & 0 & 0 & 0 & \\
\hline PIK3CA & 18 & 44 & 6 & $\mathbf{0}$ & $\mathbf{0}$ & 68 \\
\hline Exon 2 & 2 & 2 & 0 & 0 & 0 & \\
\hline Exon 5 & 0 & 3 & 0 & 0 & 0 & \\
\hline Exon 6 & 0 & 0 & 4 & 0 & 0 & \\
\hline Exon 7 & 2 & 9 & 2 & 0 & 0 & \\
\hline Exon 8 & 0 & 4 & 0 & 0 & 0 & \\
\hline Exon 10 & 12 & 12 & 0 & 0 & 0 & \\
\hline Exon 21 & 2 & 13 & 0 & 0 & 0 & \\
\hline C-KIT & 8 & 14 & 2 & $\mathbf{0}$ & 11 & 35 \\
\hline Exon 10 & 4 & 10 & 2 & 0 & 3 & \\
\hline Exon 11 & 4 & 4 & 0 & 0 & 8 & \\
\hline PDGFRA & 4 & 8 & $\mathbf{0}$ & $\mathbf{0}$ & 6 & 18 \\
\hline Exon 3 & 2 & 1 & 0 & 0 & 6 & \\
\hline Exon 12 & 2 & 7 & 0 & 0 & 0 & \\
\hline Exon 13 & 0 & 0 & 0 & 0 & 0 & \\
\hline RAF1 & $\mathbf{0}$ & 0 & $\mathbf{0}$ & $\mathbf{0}$ & $\mathbf{0}$ & $\mathbf{0}$ \\
\hline ERBB2 & 4 & 9 & 4 & $\mathbf{0}$ & $\mathbf{0}$ & 17 \\
\hline Exon 8 & 0 & 2 & 1 & 0 & 0 & \\
\hline Exon 17 & 2 & 7 & 3 & 0 & 0 & \\
\hline Exon 20 & 2 & 0 & 0 & 0 & 0 & \\
\hline ERBB3 & $\mathbf{0}$ & 2 & 0 & $\mathbf{0}$ & 0 & 2 \\
\hline Exon 3 & 0 & 2 & 0 & 0 & 0 & \\
\hline ALK & $\mathbf{0}$ & $\mathbf{0}$ & $\mathbf{0}$ & $\mathbf{0}$ & $\mathbf{0}$ & $\mathbf{0}$ \\
\hline ESR1 & $\mathbf{0}$ & $\mathbf{0}$ & $\mathbf{0}$ & $\mathbf{0}$ & $\mathbf{0}$ & $\mathbf{0}$ \\
\hline TOTAL & 110 & 171 & 34 & 13 & 17 & 345 \\
\hline
\end{tabular}

\section{Discussion:-}

NGS is a product of advanced technologies and its use has become significantly common recently and currently, this method is also used in several fields other than medicine (Goodwin S, et al., 2016,) Genome-wide sequencing has been made possible with NGS, which has started to be implemented into the routine practice in several fields of medicine including in vitro fertilization, diagnostic processes of various neurological and congenital diseases, and especially in the management of patients with cancer (Kamps R, et al. 2017). It allows for demonstrating the already 
defined mutations in several types of cancer, as well as identifying novel mutations. Identification of mutation types predicts whether a patient with an oncological disease will benefit from personalised treatments, which are increasingly becoming more common in oncology. Development of targeted drugs is on the rise especially for the treatment of CRC and NSCLC, which are the types of cancer commonly observed worldwide. However, some patients have been found to be unresponsive to the treatment despite the demonstration of respective mutations. It is obvious that additional indicators are needed for predicting the treatment response better in these patients. Identifying other mutations existing concomitantly with the already established ones may enable the treatment of these patients to be more effective.

It has been noted that $71.8 \%$ of the patients included in our study had more than one type of mutation. It has already been established that additional mutations are involved in the development of cancer. However, the capacity of newly identified mutations in predicting the treatment and prognosis of several types of cancer will be demonstrated with the contribution of study findings involving NGS. It is also recognised that regionally different types of mutations exist in some cancer types (Singh RR,et al.,2014, Susswein LR, et al., 2016). It is obvious that the presence of regionally different types of mutations is of significant importance in deciding the type of targeted treatment for these patients. Despite the low number of patients and less diversity of the cancer types, our study has introduced the simultaneous identification of regional mutations based on the NGS results in patients with cancer. Having been recently introduced for use in our country, including NGS technique in the evaluation of patients will make it possible to demonstrate the mutation profile in several types of cancer in the near future. There are many studies in the literature on the use of NGS panels in various types of cancer (Lawrence MS, et al 2013, Tsongalis GJ, et al. 2014, Schwaederle M, et al. 2015, Del Vecchio F, et al., 2017). The results of our study are compatible with those of the studies in the literature, demonstrating the frequencies and types of mutations in CRC and NSCLC (D’Haene N, et al., 2018, Cancer Genome Atlas Research Network, 2014). Demonstrating similar molecular alterations in several various types of cancer, as well as identifying the additional types of mutations, will expand the horizon for future studies on carcinogenesis (Al-Kateb H., et al. 2015, Uzilov AV, et al., 2016) Based on the results of our study, it has been determined that some of the indicators in the NGS panel do not exist at all in some types of cancer. The NGS panel we used in our study was not a customised one. Therefore, the importance of designing various types of customised NGS panels for different types of cancer should be noted.

In conclusion, determining the frequency of mutations and identifying the concomitant mutations with NGS will allow for a more precise decision-making process in the diagnosis, treatment, and prognosis estimations for patients with various types of cancer. A more common use of less costly NGS allowing for sequencing millions of DNA fragments in a shorter period of time leads to the prediction that the pieces of knowledge on carcinogenesis will accumulate by time. Furthermore, despite their unknown significance yet, several other types of mutations will be identified by means of NGS besides the demonstration of already described mutations according to the type of cancer. The unresponsiveness and different courses of prognosis in patients with similar characteristics of cancer will be better estimated with the expanding circle of knowledge in the future. A common use of NGS panels will help describe various regionally different mutation profiles in the same type of cancer. With accumulating NGS results, obtained by using a more customised selection of the indicators in the panels, a more effective use of this technique will be possible. Evaluation of the NGS results from further large-scale studies is warranted so that a better insight into carcinogenesis will be gained, types and frequencies of mutations will be defined more comprehensively by the type of cancer, and the association of these mutations with the diagnosis, treatment, and prognosis of cancer will be more precisely determined.

\section{Conflict of Interest:-}

No conflict of interest was declared by the author.

\section{Financial Disclosure:-}

The author declared that this study has received no financial support.

\section{References:-}

1. Al-Kateb H, Nguyen TT, Steger-May K, Pfeifer JD (2015). Identification of major factors associated with failed clinical molecular oncology testing performed by next generation sequencing (NGS). MolOncol 9:1737-1743.

2. Cancer Genome Atlas Research Network (2014). Comprehensive molecular profiling of lung adenocarcinoma. Nature 511: 543-550. 
3. Damodaran S, Berger MF, Roychowdhury S (2015) Clinical tumor sequencing: Opportunities and challenges for precision cancer medicine. Am SocClinOncolEducBook e175-e182.

4. Del Vecchio F, Mastroiaco V, Di Marco A, Compagnoni C, Capece D, Zazzeroni F, Capalbo C, Alesse E, Tessitore A,(2017).Next-generation sequencing: recent applications to the analysis of colorectal cancer. J Transl Med 15:246:1-19.

5. D'Haene N, Fontanges Q, De Nève N, Blanchard O, Melendez B, Delos M, Dehou MF, Maris C, et al. (2018).Clinical application of targeted next-generation sequencing for colorectal cancer patients: a multicentric Belgian experience. Oncotarget 9; 29: 20761-20768.

6. Goodwin S, McPherson, JD, McCombie, WR, (2016). Coming of age: Ten years of next-generation sequencing technologies. Nat Rev Genet 17, 333-351.

7. Kamps R, Brandão RD, van den Bosch BJ, Paulussen ADC, Xanthoulea S, Blok MJ, Romano A (2017). NextGeneration Sequencing in Oncology: Genetic Diagnosis, Risk Prediction and Cancer Classification. Int J MolSci 18, 308;1-57.

8. Koitzsch U, Heydt C, Attig H, Immerschitt I, Merkelbach-Bruse S, Fammartino A, Büttner RH, Kong Y, Odenthal M (2017). Use of the GeneReader NGS System in a clinical pathology laboratory: a comparative study. J ClinPathol 70:725-728.

9. Lawrence MS Stojanov P, Polak P, Kryukov GV, Cibulskis K, Sivachenko A, Carter SL,

10. Stewart C, Mermel CH, Roberts SA, et al. (2013) Mutational heterogeneity in cancer and the search for new cancer-associated genes. Nature 499: 214-218.

11. Schwaederle M, Daniels GA, Piccioni DE, Fanta PT, Schwab RB, Shimabukuro KA, Parker BA, Kurzrock R (2015). On the Road to Precision Cancer Medicine: Analysis of Genomic Biomarker Actionability in 439 Patients. Mol Cancer Ther 14: 1488-1494.

12. Singh RR, Patel KP, Routbort MJ, Aldape K, Lu X, Manekia J, Abraham R, Reddy NG, Barkoh BA, Veliyathu J, et al. (2014) Clinical massively parallel next-generation sequencing analysis of 409 cancer-related genesfor mutations and copy number variations in solid tumours. Br J Cancer 111: 2014-2023.

13. Susswein LR, Marshall ML, Nusbaum R, Vogel Postula KJ, Weissman SM, Yackowski L, Vaccari EM, Bissonnette J, Booke JK, Cremona ML, et al. (2016). Pathogenic and likely pathogenic variant prevalence among the first 10,000 patients referred for next-generation cancer panel testing. Genet Med 18, 823-832.

14. Tsongalis GJ, Peterson JD, de Abreu FB, Tunkey CD, Gallagher TL, Strausbaugh LD, Wells WA, Amos CI (2014) Routine use of the Ion Torrent AmpliSeq Cancer Hotspot Panel for identification of clinically actionable somatic mutations. ClinChem Lab Med 52: 707-714.

15. Uzilov AV, Ding W, Fink MY, Antipin Y, Brohl AS, Davis C, Lau CY, Pandya C, Shah H, Kasai Y, et al. (2016). Development and clinical application of an integrative genomic approach to personalized cancer therapy. Genome Med 8: 62.

16. Yu B, O'Toole SA, Trent RJ (2015). Somatic DNA mutation analysis in targeted therapy of solid tumours. TranslPediatr 2015 4: 125-138. 